\title{
Excess of radiation burden for young testicular cancer patients using automatic exposure control and contrast agent on whole-body computed tomography imaging
} \author{
Hannu Järvinen ${ }^{5}$, Eeva Salminen ${ }^{3,5}$ \\ ${ }^{1}$ Department of Medical Physics, Turku University Hospital, Turku, Finland \\ 2 Department of Diagnostic Radiology, University of Turku, Turku, Finland \\ ${ }^{3}$ Oncology and Radiotherapy, Turku University Hospital, Turku, Finland \\ ${ }^{4}$ Department of Biostatistics, University of Turku, Turku, Finland \\ ${ }_{5}^{5}$ STUK-Radiation and Nuclear Safety Authority, Helsinki, Finland
}

Hannele Niiniviita ${ }^{1,2}$, Jarmo Kulmala1,3, Tuukka Pölönen ${ }^{4}$, Heli Määttänen ${ }^{1}$,

Radiol Oncol 2017; 51(2): 235-240

Received 28 July 2016

Accepted 3 February 2017

Correspondence to: Eeva Salminen, STUK, Radiation and Nuclear Safety Authority, Laippatie 4, Fi-00880 Helsinki, Finland. Phone: +358975988752; E-mail: eevsal@utu.fi

Disclosure: The authors of this manuscript declare no relationships with any companies, whose products or services may be related to the subject matter of the article.

Background. The aim of the study was to assess patient dose from whole-body computed tomography (CT) in association with patient size, automatic exposure control (AEC) and intravenous (IV) contrast agent.

Patients and methods. Sixty-five testicular cancer patients (mean age 28 years) underwent altogether 279 wholebody CT scans from April 2000 to April 2011. The mean number of repeated examinations was 4.3. The GE LightSpeed 16 equipped with $A E C$ and the Siemens Plus 4 CT scanners were used for imaging. Whole-body scans were performed with (216) and without (63) IV contrast. The ImPACT software was used to determine the effective and organ doses. Results. Patient doses were independent $(p<0.41$ ) of patient size when the Plus 4 device (mean $7.4 \mathrm{mSv}$, SD $1.7 \mathrm{mSv}$ ) was used, but with the LightSpeed 16 AEC device, the dose (mean $14 \mathrm{mSv}$, SD $4.6 \mathrm{mSv}$ ) increased significantly $(p<0.001)$ with waist cirfumference. Imaging with the IV contrast agent caused significantly higher (13\% Plus 4, 35\% LightSpeed 16) exposure than non-contrast imaging ( $p<0.001)$.

Conclusions. Great caution on the use of IV contrast agent and careful set-up of the AEC modulation parameters is recommended to avoid excessive radiation exposure on the whole-body $\mathrm{CT}$ imaging of young patients.

Key words: automatic exposure control; computed tomography; contrast agent; radiation exposure; waist circumference

\section{Introduction}

The use of computed tomography (CT) as a diagnostic tool has increased in the past decades and nowadays CT imaging contributes most to the increase in radiation exposure of all medical radiation applications. ${ }^{1}$ Increased CT use has resulted in growing rates of repeat or multiple imaging in various patient populations and risks from cumu- lative radiation exposure have recently received more attention.,3 Some patients may go through many CT studies during the treatment and followup and they may have a long life expectancy so the associated risk from imaging should be kept as low as reasonably.

One way to reduce the overall radiation dose and to lower the cumulative dose is to reduce the dose in individual patients. ${ }^{1}$ All CT manufacturers 
have introduced online tube current output modulation systems, also known as automatic exposure control (AEC), with the main intent to decrease radiation dose without compromising image quality. These devices modulate the tube-current output in the $\mathrm{x}^{-}, \mathrm{y}^{-}$, , and $\mathrm{z}$-directions to maintain a given image noise level appropriate for patient size and volume. Indeed, automatic exposure control algorithms do reduce radiation doses by adjusting tube-current to patient size. ${ }^{4,5}$ However, the scanners without AEC were long in use together the newer devices. On these scanners consideration of patient size mainly depended on the experience and competence of the personnel, and radiation exposure parameters were adjusted only just before the examination.

The cancer patients were mainly studied with two different scanners in our hospital, so we sought to clarify how a device equipped with AEC affects the exposure to radiation of patients with different waist circumferences compared to a nonAEC device.

\section{Patients and methods}

The LightSpeed 16 (GE, Wisconsin, United States) and Plus 4 (Siemens, Erlangen, Germany) devices are third-generation CT scanners and they allow helical scanning. The LightSpeed 16 is a multi-slice CT with an adaptive array detector consisting of 24 parallel rows of solid-state detectors. The detectors cover $20 \mathrm{~mm}$ in the $\mathrm{z}$-direction at the iso-center. Detectors allow imaging of 16 slices per rotation and 0.63 to $10 \mathrm{~mm}$ slices can be reconstructed in the helical mode, depending on the reconstruction method and the selected pitch. The LightSpeed 16 device has an automatic exposure control, which adjusts the tube current to patient size and along the $\mathrm{z}$-axis, but not during rotation. The input value of AEC was the noise index. The Plus 4 device is a single-slice CT with a ceramic detector covering $10 \mathrm{~mm}$ in the $\mathrm{z}$-direction at the iso-center. The Plus 4 device can reconstruct 1 to $10 \mathrm{~mm}$ slices. It has no automatic tube current control. The main difference between the devices is in current $(\mathrm{mA})$ applications. The Plus 4 uses mainly a current of 150 $\mathrm{mA}$ for all patients, but the LightSpeed 16 exploit a wide variation of current (53 to $441 \mathrm{~mA}$ ) and the baseline is higher. Usually, a voltage of $120 \mathrm{kV}$ was used on both scanners. The Plus 4 used two series with intravenous (IV) contrast and the LightSpeed 16 examined thorax, liver and abdomen separately in order to have better dose modulation, but there were no other differences on image parameters, when IV contrast agent was used.

The study group consisted of 65 patients who underwent whole-body scanning with the two most frequently used scanners at the Department of Radiology, Turku University Hospital between the years $2000-2011$. The procedures followed Helsinki declaration and the study was approved by the South-Western Finland Hospital district's Ethical Committee.

The inclusion criteria were testicular cancer and age under 40 years. During this period this group of patients underwent 279 whole-body CT scans, on average 4.3 per patient. IV contrast agent was used on $77.4 \%$ of scans. The scanned area usually covered the whole-body from lower neck to the symphysis or mid-thigh; in a few cases it started from the external auditory canal to cover the entire neck.

Details of the imaging studies patients were obtained from the institutional radiology database. The CT-data were collected from each examination for calculation of effective doses and the patientspecific organ doses, where the doses of stomach, urinary bladder, breast, liver, red bone marrow, testicles, colon, lenses, pancreas, lungs and heart were collected. For this, software developed by ImPACT, which uses the NRPB Monte Carlo dose data sets (report SR250), was used. ${ }^{6}$ The tissueweighting factors from ICRP 103 (2007) were used to calculate the effective dose. ${ }^{7}$ For the calculations the software used voltage, current, rotation time, pitch and scanning length for input, and also tabulated the CTDI ${ }_{\text {air }}$-values, which were dependent on the scanner, voltage and collimation. The patient exposure from the LightSpeed 16, which uses current modulation, was calculated using the highest and lowest current values; the mean of these was then calculated and assumed to be closest to the actual value. The dose calculation has been described in detail by Salminen et al. ${ }^{8}$

The waist circumference was measured from one axial CT image with a metric tool (PACS, Carestream Health Inc, New York, USA). The measurement was made at the midpoint between the lowest rib and the iliac crest; the midpoint was identified visually with topogram.

Means and standard deviations (SD) or medians and range of values were used to describe continuous variables. Observations were plotted in a scatter plot and regression lines were created to illustrate the difference between scanners. For nonnormally distributed variables group differences were tested with Wilcoxon's Two-Sample Test. 


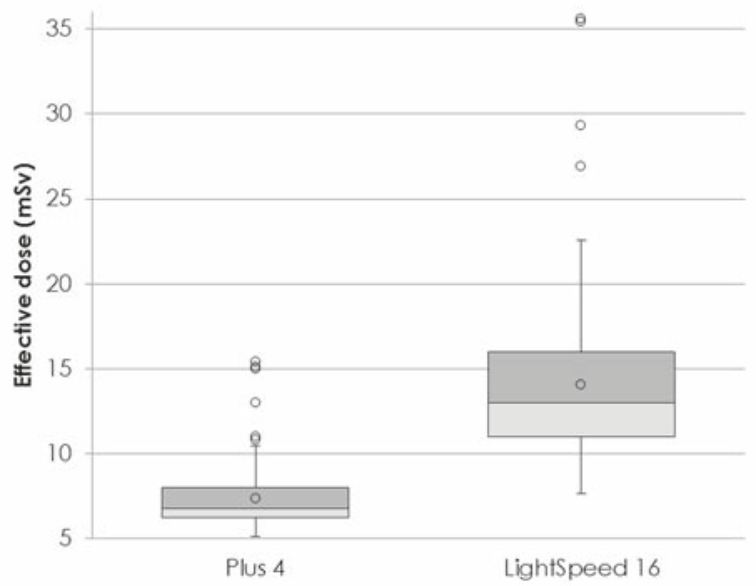

FIGURE 1. Boxplot of effective dose by device. The difference between devices is highly significant ( $p<0.0001$, Wilcoxon's).

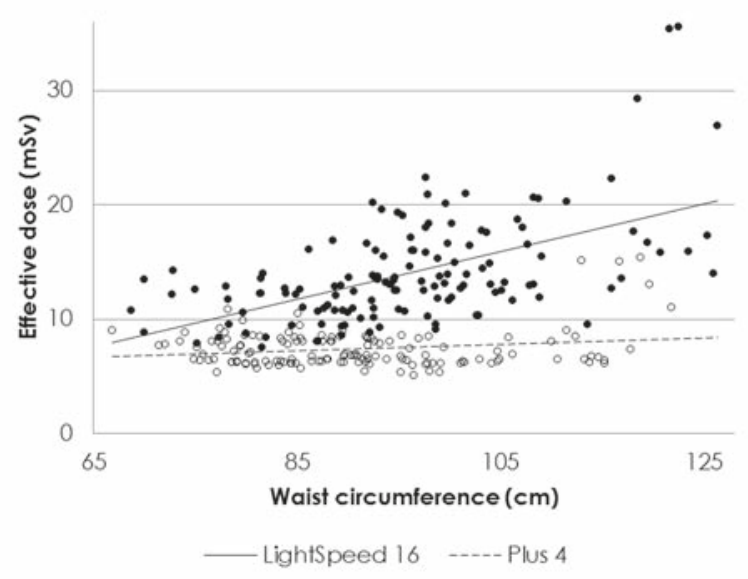

FIGURE 2. Effective dose versus waist circumference by scanner type in whole-body examinations. A wider variation of effective doses is observed for the LightSpeed 16 device.

P-values less than 0.05 were considered statistically significant. The SAS system for Windows, Version 9.3 (SAS Institute Inc, Cary, NC, USA) was used for the statistical calculations.

\section{Results}

The mean (SD) age of the patients was 28.3 (6.6) years. Further patient characteristics and number of CTs are presented in Table 1. Figure 1 shows a boxplot comparing effective doses by device. Dose levels were lower and the dose range narrower for the device without an AEC system (Plus 4). The average effective dose delivered was significantly lower for the Plus 4 than the LightSpeed $16(\mathrm{p}<$ $0.0001)$.
TABLE 1. Patient characteristics at the time of the first whole-body scan

\begin{tabular}{lc}
\hline Characteristic & Value \\
\hline Number of patients & 65 \\
Median age (years) & 28 (range 16.5-39.4) \\
Number of patients with seminoma/non-seminoma & $26 / 39$ \\
Median waist circumference (cm)† & 92.2 (range 66.9-126.3) \\
Total number of whole-body CTES & 279 \\
Mean number of whole-body CTEs/patient & $4.3($ SD 2.6) \\
Number of whole body CTEs & $137(49.1 \%)$ \\
-LightSpeed 16 & $142(50.2 \%)$ \\
-Plus 4 & $104(75.9)$ \\
Number of whole-body CTE with IV contrast & $112(78.9)$ \\
- - Plugh 4 &
\end{tabular}

† The waist circumference was measured midway between the lowest rib and the iliac crest from one exial CT image.

CTE = computed tomography examinations

TABLE 2. Mean effective dose and SD (mSv) by device and waist circumference with and without IV contrast

\begin{tabular}{lcccc}
\hline & \multicolumn{2}{c}{ LightSpeed 16* } & \multicolumn{2}{c}{ Plus 4** } \\
\hline Waist circumference & $<100 \mathrm{~cm}$ & $\geq 100 \mathrm{~cm}$ & $<100 \mathrm{~cm}$ & $\geq 100 \mathrm{~cm}$ \\
With IV & $13.6(3.2)$ & $17.5(6.1)$ & $7.4(1.2)$ & $8.5(3.3)$ \\
Without IV & $10.7(2.0)$ & $12.8(1.9)$ & $6.2(0.5)$ & $7.7(2.5)$ \\
All & $12.8(3.2)$ & $16.7(5.9)$ & $7.2(1.2)$ & $8.2(3.0)$ \\
\hline
\end{tabular}

* The p-value (Wilcoxon two-sample test) between waist circumference over and under $100 \mathrm{~cm}$ $\mathrm{p}=0.003$

** The p-value (Wilcoxon two-sample test) between waist circumference over and under $100 \mathrm{~cm}$ $p=0.003$

When the effective dose was plotted against the in waist circumference (Figure 2) a wider variation was observed for the LightSpeed 16 device, showing that patients with larger waist circumference were exposed to higher doses $(p<0.0001)$. There was a positive association between waist circumference and the dose. The effective dose generated by the LightSpeed 16 device for patients with a waist circumference $<100 \mathrm{~cm}$ was $12.8 \mathrm{mSv}$ (SD 3.2) and $>100 \mathrm{~cm} 16.7 \mathrm{mSv}$ (SD 5.9). With Plus 4 the regression analysis showed that there was no overall statistically significant change in effective dose as the waist circumference increased $(p=0.41)$. However, at a cut point of $100 \mathrm{~cm}$, there was a difference: the effective dose was $7.2 \mathrm{mSv}$ (SD 1.2) when the waist circumference was $<100 \mathrm{~cm}$ and $8.2 \mathrm{mSv}$ (SD 3.0) for $>100 \mathrm{~cm}(\mathrm{p}<0.025)$.

Table 2 shows the mean effective doses by device and waist circumference for imaging stud- 
ies with and without IV contrast. The use of IV contrast agent resulted in significantly higher radiation exposure compared to imaging studies with no IV contrast ( $p=0.002$ Plus $4, p<0.003$ LightSpeed 16).

The organ doses varied between 0.006 and 65 mGy; the lenses were subjected to the lowest dose, the stomach to the highest. Using LightSpeed 16 device the organ doses tended to increase in pace with the effective doses. For larger patients significantly higher organ doses were received by the breast, lungs, heart, stomach, liver, pancreas, colon, bladder, testicles and red bone marrow, and the doses increased statistically significantly in relation with the waist circumference for imaging studies performed with the LightSpeed 16 device ( $p<0.001$ for all organs). Such an increase in organ doses was not observed for the Plus 4 device.

\section{Discussion}

In this study, the effective dose to patients who were imaged because of testicular cancer was, on average, $7 \mathrm{mSv}$ for the Plus 4 device and $12 \mathrm{mSv}$ for the LightSpeed 16 device. The doses were significantly lower for all patients who were imaged with the older device without AEC. Use of the AEC increased the overall exposure, but it varied by patient size: smaller patients received lower doses than larger patients. The principle of AEC is to modify the current for variations in patient size. Still, the baseline level of exposure from the AEC CT-device exceeded the level of non-AEC device, and the exposure was especially high among patients of larger waist circumference. Probably the baseline reset was left on an unnecessarily high level, since the lower level of the non-AEC device was sufficient for producing proper diagnostic results.

Patients, whose waist circumference was over $100 \mathrm{~cm}$, received higher dose on LightSpeed 16, because the scanner used higher current. When the waist circumference increased over $118 \mathrm{~cm}$, the device used the maximum current during the whole examination and the current was not modulating. On LightSpeed 16 the voltage remain unchanged $(120 \mathrm{kV})$ regardless of waist circumference. The reason for higher doses on Plus 4 was the higher voltage $(140 \mathrm{kV})$, which was used, when the waist circumference was over $113 \mathrm{~cm}$.

Comparing procedures performed by devices it should be noted that also the number of series were different. On Plus 4 there were two series, when IV contrast was used while there were three series on LightSpeed 16. The LightSpeed 16 examined thorax, liver and abdomen separately in order to have better dose modulation. This contributes to higher patient exposure on LightSpeed 16. Basic set up level for LightSpeed 16 was also high, perhaps following the vendor's recommendation rather than being adapted to a departmental protocol.

Based on data available on how patient biometrics should be considered in CT imaging. ${ }^{9,10,11}$ Chan and associates have addressed the question of BMI and abdominal fat. They found that by increasing these variables effective doses from the abdomen and pelvis scans also significantly increased. ${ }^{10}$ They observed a potential risk of very high radiation doses to oversized patients when the automatic exposure control system is used. Our results are in concert with this and point out the importance of careful consideration of precise values. A study by Kalra et al. ${ }^{12}$ reported that a z-axis modulating AEC, if used correctly, can reduce the dose by 34$45 \%$. Optimum use requires an understanding of the importance to reset parameters and of the effect of IV contrast agent on radiation exposure.

The noise index (NI) was used as an input factor in the AEC of the LightSpeed 16 device. The NI is approximately equal to the SD of reconstructed images and allows selecting the amount of noise of images. The NI determines the tube current within the selected range. In our study a higher tube current was used in the LightSpeed 16 device, which implies that the NI was set too low and especially larger patients require selection of a higher NI, because more noise can be moderated on larger patients and a 5\% increase in NI is associated with a $10 \%$ reduction in radiation dose. ${ }^{13}$ There are also another means to lower the dose in addition to increasing the NI, since all parameters that affect image noise affect indirectly the tube current when an AEC is used. ${ }^{14}$

Use of IV contrast increased the radiation dose from both devices further. Paul and associates observed that CTs done with IV contrast agent raises the dose in chest imaging in AEC-equipped CTs. ${ }^{15}$ The use of IV contrast agent usually involves multiple imaging steps: first native CT and then contrast-augmented CT. More phases naturally increase the effective dose to the patient. The use of IV contrast agent does not always provide additional diagnostic information and the usefulness of doing both a native and a contrast $\mathrm{CT}$ requires further study. ${ }^{16,17,18}$

The use of already used or refurbished CT scanners is becoming more popular in less privileged 
countries. Concerns regarding the poor quality of these devices have been expressed..$^{19}$ We voice our concern also for the lag of appropriate and timely maintenance and for the understanding of the importance of protocol details, regardless of the type or age of the device. Even older devices could be used achieving sufficient diagnostic value without increasing patient exposure unnecessarily.

The advantages of the current study include the use of 1-slice and 16-slice CT devices in the same hospital environment. We could collect all data comprehensively since all patients were followed up in the same hospital and scanning was exclusively performed with only two CT devices. The disadvantage of this study was the old fashion devices. However, this is understandable, while the examinations have been done since 2000, when the 16-slice scanners were the state of the art. While 64-slice scanners or more slice scanners are nowadays widely used, it would also be interesting to study the differences between patients with newer devices.

There were significant differences in the amount of radiation exposure to the patients scanned with these two devices. Thus, it is necessary to understand the technical characteristics of each CT device in addition to the scanning protocol, when radiation exposure is determined clinically. The protocol should be adapted to patient biometrics: exposure may be increased only when more radiation is required to improve the quality of the scans. In all clinical radiology, unnecessary exposure must be avoided. Imaging records and protocols should describe in detail when it is allowable to deviate from the pre-assigned imaging protocol regarding radiation dose and use of IV contrast agent. This information is needed for clinical reasons, but importantly also for purposes of reconstructing the estimated amount of radiation exposure to the individual.

Although all imaging studies were done in one hospital, we could not retrospectively establish the reason for why the basic dose levels were higher also for small patients, when the AEC was used compared to previous practice. The reason may reside in the vendor's recommendation. If so, this stands in contrast to the principle of using an AEC: to provide a substantial reduction in radiation dose with similar or improved image quality. ${ }^{20}$ As the image quality of LightSpeed 16 may have exceeded the image quality needed for clinical decision, there is a need for image quality assessment between these two devices to further explain the differences in doses.

\section{Conclusions}

The current results show that there is a need for careful consideration of the set-up of the basic parameters for AEC-equipped CT devices. This need stems from the present observation that patient size and the use of IV contrast media are associated with an excessive risk of unnecessarily high radiation exposure. Proper attention to these circumstances is warranted for improved radiation protection in connection with CT-imaging studies.

\section{Acknowledgements}

The study was supported by a government research grant (EVO Foundation) awarded to Turku University Hospital.

\section{References}

1. Brenner DJ, Hall EJ. Computed tomography - an increasing source of radiation exposure. N Engl J Med 2007; 357: 2277-84. doi: 10.1056/ NEJMra072149

2. Griffey RT, Sodickson A. Cumulative radiation exposure and cancer risk estimates in emergency department patients undergoing repeat or multiple CT. AJR Am J Roentgenol 2009; 192: 887-92. doi:10.2214/AJR.08.1351

3. Brenner DJ, Doll R, Goodhead DT, Hall EJ, Land CE, Little JB, et al. Cancer risks attributable to low doses of ionizing radiation: Assessing what we really know. Proc Natl Acad Sci U S A 2003; 100: 13761-6. doi:10.1073/ pnas. 2235592100

4. Ghoshhajra BB, Engel LC, Károlyi M, Sidhu MS, Wai B, Barreto M, et al. Cardiac computed tomography angiography with automatic tube potential selection: effects on radiation dose and image quality. J Thorac Imaging 2013; 28: 40-8. doi: 10.1097/RTI.0b013e3182631e8a

5. Lee $\mathrm{K}$, Lee $\mathrm{W}$, Lee J, Lee $\mathrm{B}, \mathrm{Oh} \mathrm{G}$. Dose reduction and image quality assessment in MDCT using AEC (D-Dom § Z-Dom) and in-plane bismuth shielding. Radiat Prot Dosim 2010; 141: 162-7. doi: 10.1093/rpd/ncq159

6. Shrimpton PC, Jones DG. NRPB-SR250: Normalised organ doses for $\mathrm{x}$-ray computed tomography calculated using Monte Carlo techniques. Radiat Prot Dosim 1993; 49: 241-3.

7. International Commission on Radiological Protection. The 2007 Recommendations of the International Commission on Radiological Protection. ICRP Publication 103 2002; 37 (2-4).

8. Salminen $E$, Niiniviita $H$, Kulmala J, Määttänen $H$, Järvinen $H$. Radiation dose estimation in computed tomography examinations using NRPB-SR250 software in a retrospective analysis of a patient population. Radiat Prot Dosim 2012; 152: 328-33. doi: 10.1093/rpd/ncs065

9. Sodickson A, Weiss M. Effects of patient size on radiation dose reduction and image quality in low kVp CT pulmonary angiography performed with reduced IV contrast dose. Emerg Radiol 2012; 19: 437-45. doi: 10.1007/ s10140-012-1046-z

10. Chan VO, McDermott S, Buckley O, Allen S, Casey M, O'Laoide R, et al. The relationship of body mass index and abdominal fat on the radiation dose received during routine computed tomographic imaging on the abdomen and pelvis. Can Assoc Radiol J 2012; 63: 260-6. doi: 10.1016/j.carj.2011.02.006

11. Alkadhi H, Stolzmann P, Scheffel H, Desbiolles L, Baumüller S, Plass A et al. Radiation dose of cardiac dual-source CT: the effect of tailoring the protocol to patient-specific parameters. Eur J Radio/ 2008; 68: 385-91. doi: 10.1016/j. ejrad.2008.08.015 
12. Kalra MK, Maher MM, Toht TL, Kamath RS, Halpern EF, Saini S. Comparison of Z-axis automatic tube current modulation technique with fixed tube current CT scanning of abdomen and pelvis. Radiology 2004; 232: 347-53. doi: 10.1148/radiol.2322031304

13. Kalra MK, Naz N, Rizzo S, Blake MA. Computed tomography radiation dose optimization: scanning protocols and clinical applications of automatic exposure control. Curr Probl Diagn Radiol 2005; 34: 171-81. doi: 10.1067/j. cpradiol.2005.06.002

14. Gudjónsdóttir J, Ween B, Olesn DR. Optimal use of AEC in CT: A literature review. Radiol Tech 2010; 81: 309-17.

15. Paul J, Schell B, Kerl JM, Maentele W, VogI TJ, Bauer RW. Effect of contras material on image noise and radiation dose in adult chest computed tomography using automatic exposure control: a comparative study between 16-,64- and 128-slice CT. Eur J Radiol 2011; 79: 28-32. doi: 10.1016/j. ejrad.2011.05.012

16. da Costa e Silva EJ, da Silva GA. Elimination unenhanced CT when evaluating abdominal neoplasms in children. AJR Am J Roentgenol 2007; 189: 1211-14. doi:10.2214/AJR.07.2154

17. Patten RM, Byun JY, Freeny PC. CT of hypervascular hepatic tumours: are unenhanced scans necessary for diagnosis. AJR Am J Roentgenol 1993; 161: 979-84. doi: 10.2214/ajr.161.5.8273641

18. Jung SI, Park HS, Kim YJ, Jeon HJ. Multidetector computed tomography for the assessment of adnexal mass: Is unenhanced CT scan necessary. Korean J Radiol 2014; 15: 72-9. doi: 10.3348/kjr.2014.15.1.72

19. Hong JS, Kang HC. Relationship between the use of new or used computed tomography scanners and image retake rates in South Korea. Acta Radio 2013; 54: 428-34. doi: 10.1258/ar.2012.120290

20. Tack D, De Maertelaer V, Gevenois PA. Dose reduction in multidetector CT using attenuation- based online tube current modulation. AJR Am J Roentgenol 2003; 181: 331-4. doi: 10.2214/ajr.181.2.1810331 